\title{
La objeción de conciencia en la práctica clínica
}

\author{
Adela Montero Vega ${ }^{1}$, Electra González Araya ${ }^{2}$
}

Resumen: Se describe la objeción de conciencia en la práctica clínica como un derecho de los profesionales, fundamentado principalmente en la libertad de conciencia. Se realiza revisión bibliográfica abarcando los últimos diez ańos para conocer su real significado, magnitud y trascendencia. Se concluye que debe plantearse en situaciones específicas, como una excepción, con prudencia, compatibilizando los derechos de todos los involucrados, alcanzando el equilibrio en nuestras decisiones, permitiendo que una sociedad donde conviven personas con diferentes valores y creencias aspiren en conjunto al bien común, condición necesaria para fortalecer la democracia.

Palabras clave: conciencia, objeción de conciencia, bioética, ética médica

\section{Conscience objection in clinical practice}

\begin{abstract}
Conscience objection is described in clinical practice as a professional right, based mainly in freedom of conscience. A bibliographical review of last ten years is carried out to know the real meaning, extent and transcendence. The conclusion is that it must raise in specific situations, as an exception, with prudence, harmonizing the rights of all participating, reaching equilibrium in decision making, allowing that a society where persons with different values and beliefs live together aspire overall to common good, necessary condition to strengthen democracy
\end{abstract}

Key words: conscience, conscience objection, bioethics, medical ethics

\section{A objeção de consciência na prática clínica}

Resumo: Descreve-se a objeção de consciência na prática clínica como um direito dos profissionais fundamentado principalmente na liberdade de consciência. Realiza-se uma revisão bibliográfica abarcando os últimos 10 anos para conhecer seu real significado, magnitude e transcendência. Conclui que se deve apresentá-la em situaçóes específicas, como uma exceção, com prudência, compatibilizando os direitos de todos os envolvidos, alcançando o equilíbrio em nossas decisóes, permitindo que uma sociedade onde convivem pessoas com diferentes valores e crenças aspirem em conjunto o bem comum, condição necessária para fortalecer a democracia.

Palavras-chave: consciência; objeção de consciência; bioética; ética médica

\footnotetext{
${ }^{1}$ Médico Gineco-Obstetra. Profesor Asistente, Facultad de Medicina, Universidad de Chile, Chile. Correspondencia: amontero@med.uchile.cl

${ }^{2}$ Asistente Social. Master en Estudios de Población, Universidad de Exeter. Profesor Asociado, Facultad de Medicina, Universidad de Chile, Chile
} 


\section{Introducción}

La objeción de conciencia constituye un tema difícil y complejo, presente desde la antigüedad en la discusión ética(1), pues alude a la dignidad humana basada en los dictámenes de la conciencia, llegándose a plantear que debería ser considerada como un derecho fundamental de las personas, de rango constitucional(2). Sin embargo, en la práctica clínica, el problema radica, por una parte, en el legítimo derecho del individuo objetor a la libertad de conciencia y, por otra, en el también legítimo derecho de las personas a recibir una atención de salud digna, de calidad y sin discriminación(3).

La observancia de la valoración y definición de la salud es un asunto que compete a todos los ciudadanos y al Estado. Para toda sociedad democrática y justa es necesario asegurar el derecho a la salud como una forma básica de convivencia, utilizando para ello mecanismos informales como la ética y la moral, así como la consideración de mecanismos debidamente formales o institucionalizados como el Derecho(4). La ética antecede al Derecho en la fundamentación de una norma jurídica y también lo sucede, verificando que el respeto y el cumplimiento de la formulación jurídica resguarde los fundamentos éticos. El Derecho, a su vez, salvaguarda los derechos de los individuos pertenecientes a una sociedad democrática, mediante la concreción de una norma jurídica, la que debe ser respetada y obedecida por todos los integrantes de las sociedades pluralistas(4). Debemos considerar la posibilidad de que el derecho a la salud y el derecho al acceso a la atención sanitaria no se vea limitado o entorpecido por una objeción de conciencia mal entendida, pudiendo llegar a ser utilizada inapropiadamente como una manera de eludir o de encubrir las responsabilidades profesionales(5), o como una forma encubierta de discriminación o utilización por parte de los profesionales en busca del propio interés(6), determinando como consecuencia un grave abuso hacia los derechos fundamentales de las personas, con el consiguiente menoscabo de la salud de la población(7).

El objetivo de esta publicación es conocer el real significado, magnitud y trascendencia de la objeción de conciencia en la práctica clínica. Para ello, es necesario considerar aspectos como qué se entiende por los conceptos de "conciencia", "conciencia moral" y "objeción de conciencia" en la atención sanitaria, y conocer los principales aspectos relacionados con la objeción de conciencia en este ámbito.

\section{Método}

Este estudio pretende ser de carácter descriptivo, considerando la perspectiva actual de la objeción de conciencia en la práctica clínica, tanto en Chile como en el extranjero. Se realizó una búsqueda bibliográfica en internet de los artículos publicados en revistas científicas indexadas durante los últimos 10 años, accediendo a PUBMED, LILACS y SciELO, para conocer el estado del arte respecto de este tema.

En el desarrollo de esta publicación revisaremos las definiciones sobre conciencia, conciencia moral, objeción de conciencia y otros términos involucrados, y la descripción global respecto de la magnitud, trascendencia, relevancia y límites de la objeción de conciencia en la práctica clínica.

\section{Resultados}

"Conciencia" es un término que deriva del latín

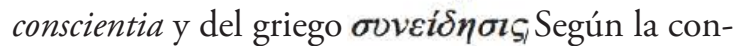
cepción habitual, encontramos diversos significados como: "aquella propiedad del espíritu humano de reconocerse en sus atributos esenciales y en todas las modificaciones que en sí experimenta”; "conocimiento reflexivo de las cosas"; "actividad mental a la que sólo puede tener acceso el propio sujeto" o "conocimiento interior del bien y del mal"(8). La Associació Catalana d' Estudis Bioètics (ACEB) alude a la conciencia como: "aquel juicio reflexivo por el que distinguimos interiormente el bien del mal, la actuación correcta de la incorrecta, la acción honesta de la deshonesta, la conducta ética y moral, de la inmoral y sin ética" (1). Sieira la considera como: "dictamen de lo que moralmente puede hacerse u omitirse en una situación concreta”, o "juicios personales sobre la moralidad de la acción singular y concreta que va a realizarse, se está realizando o se ha 
realizado"(2). Desde un punto de vista filosófico, la conciencia también es considerada desde varias acepciones(9). Una de ellas se refiere a la relación del alma consigo misma, en una relación intrínseca al hombre interior o espiritual, por medio de lo cual se puede juzgar a sí mismo para proceder a evaluar de manera inmediata y privilegiada lo correcto de lo incorrecto. De lo anterior se desprende la capacidad de autojuzgarse, parte de la "conciencia moral", que sería la relación privada del hombre consigo mismo, en la cual éste se extrapola o separa de los demás entes "retornando a sí mismo", testimoniándose a sí mismo, dando lugar a una investigación interior o introspección, que le permita llegar a conocerse en forma certera $y \operatorname{veraz}(9)$.

Esta conciencia moral aludiría a aquella condición que se entiende por innata, intrínseca al hombre o aquello que todos los hombres poseen por el sólo hecho de serlo. Para Sócrates, la conciencia moral pertenecería al "Daimon", como una forma de intervención en los momentos difíciles de la existencia humana, donde esta figura indicaría principalmente lo que se debe omitir. La concepción aristotélica hace referencia a aquello procedente del "sentido" moral. Tomás de Aquino la consideraría como el espíritu que indica si un acto es justo o no(10). Para Kant, es la razón práctica que representa al hombre su deber respecto de absolverlo o condenarlo moralmente por sus acciones(11). Para otros autores, esta forma particular de conciencia se traduciría como la percepción de las malas acciones que se han cometido o como la capacidad de juzgar el bien y el mal. La conciencia moral, al discernir entre lo bueno y lo malo, entre lo justo y lo injusto, permitiría al hombre realizar juicios sobre las acciones pasadas y tener en consideración las acciones futuras. Es concebida como el sentido moral o la facultad que posibilita la percepción de la calidad moral de nuestras acciones, lo que nos haría experimentar un sentimiento de aprobación o de condena respecto de ellas(11).

Para Juan Pablo II(12), la conciencia moral sería la capacidad de discernimiento que tiene el hombre para descubrir y obrar según la ley que Dios ha inscrito en cada hombre, la que debe conformarse en función de la verdad objetiva, universal e igual para todos, siendo una obligación de cada persona ir en búsqueda de esta verdad. El hombre, al dirigir sus actos según la propia conciencia, debe considerar no sólo este deseo por la verdad, sino también el respeto a la ley moral establecida en el hombre por Dios. Considera de vital importancia a la familia, la escuela, la educación religiosa y los medios de comunicación como aspectos fundamentales en la conformación de la conciencia de la persona(12).

La objeción de conciencia es considerada conceptualmente desde diversas posturas, con cierto grado de imprecisión. Una de las definiciones la señala como una forma de incumplimiento de una obligación de naturaleza legal, cuyo acatamiento o realización produciría en la persona una grave lesión de su conciencia, con base en motivaciones de índole ético, moral, religioso, axiológico o de justicia del individuo objetor $(6,13)$. También es entendida como un derecho subjetivo frente a una obligación o normativa legal, con incumplimiento de ésta por razones religiosas o filosóficas, derivada de las relaciones laborales o funcionarias, públicas o privadas, de un individuo(5). Sin embargo, al entender la conciencia como algo perteneciente a la esfera individual, inherente al propio ser humano, se entendería la objeción de conciencia como aquella negación vinculada al contexto privado del hombre, planteándose como una obligación personal, no pudiendo ser en ningún caso de índole colectiva ni institucional $(5,7,14)$. Otra concepción alude a la negativa por razones ideológicas o religiosas del profesional de la salud frente a instancias médicas específicas, como la práctica del aborto y la eutanasia, derivadas de un mandato proveniente desde una orden superior, administrativa o legal $(13,15)$. Domínguez plantea la objeción de conciencia como una entidad separada de la desobediencia civil, describiéndola como la negativa o rechazo, de carácter individual, ante un mandato legal o administrativo, para practicar un procedimiento por razones de índole ética, moral, religiosa u otras, por parte del personal sanitario, del paciente o incluso de sus propios familiares(15).

Desde un punto de vista sociológico, se la ha 
considerado como el conflicto producido entre la norma jurídica, que impone un deber, y la norma moral por parte del objetor, que se contrapone al cumplimiento de la anterior; en otras palabras, es la negación de obedecer un mandato de la autoridad legítimamente estatuida por considerarlo injusto o delictuoso en consideración a la normativa moral. Según este aspecto, sería una competencia de motivaciones diferentes respecto de la real normativa social imperante en una sociedad democrática(2).

Desde un punto de vista jurídico, Neira y cols., aluden a este concepto como: "la negativa a cumplir un mandato de la autoridad o una norma jurídica invocando la existencia en el fuero de la conciencia, de un imperativo que prohíbe dicho cumplimiento"(16). Para Rawls consistiría en el no consentimiento consciente, que no apela al sentido de justicia de la mayoría respecto de un mandato legislativo más o menos directo o una orden administrativa, fundada en principios políticos, de justicia, religiosos o de otra clase, por parte de una persona en una sociedad libre(17). Finalmente, según la perspectiva bioética, representaría la negativa del individuo en base a su autonomía y autodeterminación para ejecutar una acción derivada de un mandato legal, jurídico o administrativo, que altere o vulnere sus principios morales, religiosos o ideológicos $(15,16,18)$.

Es importante considerar las diferencias relacionadas con la desobediencia civil, la desobediencia al Derecho, el derecho a la resistencia, la acción militante, la objeción de ciencia y la objeción de legalidad, debido a la tendencia a considerar otras acciones como una genuina objeción de conciencia.

Domínguez concibe la desobediencia civil derivada de una dimensión social, caracterizada por una movilización colectiva, no violenta, como oposición a una norma jurídica considerada lesiva u ofensiva a los intereses de la sociedad o a los hábitos o costumbres imperantes en ella(15). Otros consideran que no cabe el reconocimiento de la desobediencia civil por parte del Derecho, por ende no podría considerársele un derecho en sí(16). La desobediencia civil busca el cambio político de una norma o ley por consideraciones políticas o ideológicas; al contrario de la objeción de conciencia, que sólo involucra al objetor, en forma individual y privada, el cual no pretende un cambio en la legalidad vigente, sino más bien su opción personal de negativa ante un mandato jurídico y/o administrativo, fundándose en la preservación de su integridad moral, ideológica o religiosa(16).

La desobediencia al Derecho implica el cuestionamiento y no acatamiento al ordenamiento jurídico imperante en un Estado en particular, con la salvedad que la motivación no es por razones de conciencia(16).

El derecho a la resistencia se funda en una oposición radical al ordenamiento jurídico imperante, oponiéndose principalmente a la fundamentación y a la finalidad de este ordenamiento, planteando la creación de un nuevo orden jurídico por considerar el actual orden como directamente opresor. Se trata de un acto público, de carácter político, con una base político-ideológica, que buscaría no cambiar una norma o ley establecida, como en la desobediencia civil, sino que el orden jurídicopolítico establecido(16).

La acción militante se refiere a aquellas acciones armadas organizadas, destinadas a oponerse a un determinado régimen político, con el propósito de desestabilizarlo, no apelando a las concepciones o principios de justicia del régimen al que se espera combatir, sino por considerarlo injusto e ilegítimo(18).

La objeción de ciencia sería la objeción invocada por razones científicas, por ejemplo, de las personas integradas en centros de trabajo, de investigación o estudio, donde las actividades desarrolladas impliquen, en el presente o en el futuro, un serio daño para el medio ambiente, los seres vivos, la dignidad y los derechos fundamentales de las personas, como lo concerniente a la investigación genética por sus potenciales implicancias éticas, legales y sociales (2).

La objeción de legalidad se referiría a aquella negativa de los profesionales para realizar acciones que vayan en contra de la legislación vigente, en las que una determinada acción presupone o pue- 
da suponer acciones constitutivas de delito(2). Un ejemplo sería la negativa a la realización de prácticas abortivas en países donde el aborto está penalizado, no por razones de conciencia, sino para evitar caer en situaciones al margen de la legalidad vigente.

Magnitud, trascendencia e implicancias de la objeción de conciencia en la práctica sanitaria

En Chile, en los últimos 10 años, encontramos escasas publicaciones científicas que aborden esta temática, encontrando sólo un artículo relacionado con la atención en salud(5). En el plano internacional, existen múltiples publicaciones en las que se aborda desde diferentes ángulos, lo que con frecuencia produce ambivalencia y confusión, representando en ocasiones un arduo debate y réplicas entre los autores(19-22). Desde principios del siglo XX, principalmente en España y otros países europeos, se ha venido observando una corriente relacionada con este tipo de objeción ante la oposición para desarrollar determinadas obligaciones, como es el servicio militar(23) y la participación directa en conflictos bélicos(15). Posteriormente surgen las primeras evidencias explícitas de objeción de conciencia sanitaria, resultando paradigmáticos los casos de profesionales objetores a diferentes instancias, como el aborto, la anticoncepción hormonal, la esterilización, la fertilización asistida, la consejería reproductiva a parejas homosexuales, a mujeres solteras y a otros grupos discriminados socialmente $(5,7,19,24)$.

A medida que el pluralismo ético de nuestras sociedades ha ido haciéndose más patente, sumado al creciente desarrollo científico-tecnológico, enfrentamos cada día nuevas causales para plantear este tipo de objeción, como el rechazo pacifista para colaborar en medicina militar(25), la negativa de los profesionales para participar en prácticas como la eutanasia, el suicidio médicamente asistido, el desarrollo de ensayos clínicos(25), el diagnóstico prenatal de enfermedades genéticas y cromosómicas(26), y la manipulación de embriones humanos (27). Otros autores describen la formulación de esta objeción en otras situaciones, como la esterilización, principalmente de personas con discapacidad psíquica, la suspensión de tratamientos médicos, la investigación destructiva de embriones, la selección preconcepcional del sexo, la participación en alimentación forzada de personas en huelga de hambre, los reconocimientos médicos para el servicio militar, la cooperación con la policía en obtención de información (interrogatorios), las transfusiones de sangre (Testigos de Jehová), el transplante de órganos, la psicocirugía y la experimentación con seres humanos y animales (28).

Es así como en la práctica clínica se tienden a producir conflictos de tensión creciente cuando se plantean situaciones relacionadas con esta materia, donde se confrontan los derechos del paciente con los derechos y deberes del profesional objetor(19). Es importante considerar que, aun cuando en el ámbito de la salud la objeción de conciencia se ha relacionado principalmente con la ejercida por parte de los profesionales médicos, también se han reportado situaciones donde otros profesionales sanitarios, que no intervienen directamente en el procedimiento por el cual se objeta en conciencia, realizan una negativa por razones de conciencia a la atención de los/las pacientes o personas que legítimamente tienen derecho a esta prestación. Se ha reportado que estos profesionales han objetado participar aún en forma indirecta en el procedimiento, incluso con negativa respecto al cuidado del paciente, rehusándose a colaborar en la preparación del instrumental médico o en el traslado del paciente a otro centro asistencial $(2,16)$. Debe destacarse que la genuina objeción de conciencia no es hacia las personas, sino hacia una acción directa específica, por lo que en ningún caso se debiera negar la asistencia necesaria como apoyo a la condición que el paciente amerita(25). Por ejemplo, un profesional objetor de conciencia al aborto puede objetar al procedimiento en sí, pero no puede negarse al cuidado médico o de colaboración médica antes o después de efectuado el procedimiento por un profesional no objetor $(7,29)$. Del mismo modo, el personal de colaboración médica que no está ejecutando la acción, no podría objetar cumplir con lo establecido en sus funciones profesionales, como sería el brindar apoyo antes y después del procedimiento(29). 
La objeción se admitiría como válida sólo cuando existe una participación directa del profesional, como sería practicar la intervención, la prescripción de medicamentos con fines abortivos o la prestación de servicios de anestesia y de enfermería durante el procedimiento(7). Por otra parte, se ha reportado la negativa del profesional farmacéutico para despachar receta por anticoncepción de emergencia a una víctima de violación(19), situaciones lamentablemente también reportadas por otros autores(16). Respecto de este punto, existe jurisprudencia en la Corte Europea de Derechos Humanos al determinar que los farmacéuticos podían manifestar su libertad de conciencia respecto a sus convicciones religiosas en la esfera de su vida privada o personal, sin negar las diligencias necesarias para proveer las prescripciones referentes a esta anticoncepción(7,30). Estas diferentes posturas han sido determinantes para que algunas organizaciones profesionales hayan establecido directrices éticas para guiar al personal sanitario respecto a la objeción de conciencia $(14,30)$, las que, si bien reconocen el derecho profesional para objetar, también reconocen el derecho de la población a recibir una atención de calidad sin riesgo para su vida y su salud, planteando alternativas que no perjudiquen a ninguna de las partes involucradas, recomendando: la derivación oportuna del paciente a un profesional no objetor, el traslado oportuno del paciente a otro centro asistencial, la no posibilidad de objetar frente a situaciones de emergencia, evitar prácticas discriminatorias, el respeto por las diferencias personales y culturales en la relación profesionalpaciente y entre los propios profesionales(30).

Desde el aspecto ético, la objeción de conciencia se basaría en la responsabilidad de la emisión prudente de un juicio ético sobre una determinada acción(27), asociada a una honestidad en la conducta y a una vida de la persona objetora coherente y consecuente con los principios que motivan la objeción(1). En este punto encontramos dos grandes corrientes: la relativista y la personalista. La primera fundamenta los principios y valores morales en una base empírico racional, apoyándose en la verificación de los hechos y en la coherencia de la argumentación, considerando en su fundamentación los principios bioéticos de autonomía, beneficencia y justicia, alcanzando en nuestras sociedades contemporáneas un valor preponderante el respeto por la autonomía. Este principio, en conjunto con el de beneficencia, constituirían el vector principal en la fundamentación de la objeción de conciencia por parte de los profesionales objetores que aluden a esta corriente bioética relativista(13). La corriente personalista, inspirada en Tomás de Aquino, se fundamenta principalmente en el carácter ontológico del respeto por la vida humana, desde el momento de la concepción y hasta el último instante de vida, fundamento sostenido por muchos objetores que recurren a la objeción de conciencia considerando los aspectos religiosos de una determinada acción(13).

Otro aspecto importante se vincula con la condición laboral del profesional sanitario objetor, considerándose éticamente reprobable que la objeción se plantee sólo en situaciones donde existe un contrato institucional, en la esfera del servicio público y no se realice cuando el mismo objetor se desempeñe en la esfera privada o en forma independiente(25). Tampoco podrá suponer para el objetor la obtención de ventajas laborales ni tampoco desventajas como posibles amonestaciones, marginación, ni algún tipo de discriminación(25). Asimismo, es necesario considerar la relación entre la objeción de conciencia y las instituciones sanitarias. Respecto de los centros asistenciales, la objeción de conciencia es una práctica relativa a la conciencia y por ende individual, no pudiendo en consecuencia las instituciones declararse ni reconocerse como objetoras. Esta condición involucra a los jefes administrativos, quienes aun cuando objeten en conciencia respecto de una determinada acción o intervención, no pueden involucrar a la unidad, servicio o centro asistencial; muy por el contrario, deben asegurar que las dependencias a su cargo cumplan con la normativa legal vigente, proporcionando a sus usuarios el mejor estándar de salud imperante en su comunidad $(7,29)$.

Límites de la objeción de conciencia en la atención clínica

Es fundamental reconocer la existencia de límites, siendo indispensable preguntarse: ¿existe como 
consecuencia de esta objeción un perjuicio directo para la vida o la salud del paciente? ¿Existe disponibilidad de que otro/a profesional ejecute o brinde la atención en salud que el paciente necesita? ¿Es factible plantear la derivación del paciente a otro profesional o a otro centro asistencial para que se le brinde la atención necesaria? ¿Qué sucederá en aquellas situaciones de urgencia en las que es imprescindible ejecutar la acción objetada para salvar la vida del paciente? ¿Qué sucederá en aquellas situaciones en las que el único profesional disponible es el profesional objetor? ¿Es deber del objetor explicitar frente al paciente o frente a su superior jerárquico su objeción de conciencia? ¿En qué momento de la atención en salud el objetor debe explicitar su objeción de conciencia? ¿Puede el objetor manipular la información e incluso ocultar parte de la información al paciente o autoridades por considerar que al hacerlo se transforma en facilitador del acto que va en contra de su conciencia? ¿Puede un profesional negarse a cumplir un acuerdo tomado por el equipo médico tratante por discrepar de la decisión? ¿Debe el profesional respetar la voluntad y autonomía del paciente aun cuando vaya en contra de su conciencia?(31,32).

Todas estas interrogantes, y posiblemente otras, se deben evaluar considerando que el derecho a la objeción de conciencia no puede ser absoluto si involucra la vida o la salud de otros(7), debiendo las decisiones involucrar el contexto en el que se desarrolla la problemática, considerando el derecho fundamental de la dignidad humana, respetando el sistema de valores, de creencias y convicciones tanto del profesional objetor, como de la o las persona (s) posiblemente afectadas. El conflicto se presenta cuando se contraponen el derecho a la libertad de conciencia y la autonomía del profesional objetor, versus el derecho a la autonomía y necesidad de atención en salud del paciente, considerando que la salud y la vida son bienes jurídicos que hay que asegurar por encima de un deber de obediencia u objeción de conciencia. El problema se hace más patente cuando esta objeción formulada por un profesional se contrapone con el también legítimo derecho del otro u otros para recibir una atención en salud que está respaldada por ley.
En general, la objeción de conciencia por parte del profesional sanitario debiera respetarse cuando se trate de una verdadera objeción, que no se confunda con una medida para imponer una determinada postura ética o religiosa, o como un acto discriminatorio hacia los pacientes o una forma de eludir responsabilidades por parte del profesional frente a hechos conflictivos. Es prioritario que bajo ninguna circunstancia se determine una acción de maleficencia o perjuicio para la vida o salud del paciente. Por otra parte, debe existir la seguridad de que otro profesional pueda resolver adecuadamente el problema de salud al que se objeta y cuya prestación se encuentra respaldada por ley. También se considera el traslado oportuno y eficaz del paciente a otro centro asistencial, siempre que las condiciones del momento lo permitan, siendo una excepción las situaciones de urgencia, en la que la gravedad de la condición clínica o las serias consecuencias en la salud del paciente, así como otras situaciones que pueden llegar a involucrar incluso la seguridad de otras personas, no permitan este traslado en forma expedita.

Respecto de los deberes del profesional, se considera que debe explicitar con anterioridad su objeción al superior jerárquico, para que la institución pueda tomar los resguardos necesarios, garantizando el acceso de la población a los mejores estándares de salud, respaldados por la legalidad vigente en esa comunidad. El objetor debiera exponer lo más precozmente posible ante su paciente las situaciones que motivarán su objeción, otorgando la libertad y facilidad para que el paciente opte por otro profesional, planteando incluso que el profesional objetor efectúe la derivación a un profesional no objetor.

\section{Discusión}

Si la objeción de conciencia se plantea como un derecho, basado en la libertad de conciencia individual de las personas, también debe reconocerse el derecho de los pacientes a una atención de salud de calidad, con respeto a su dignidad y necesidades.

Las instituciones sanitarias y académicas no pueden esgrimir indiscriminadamente el derecho a 
esta objeción, pues ésta corresponde al plano subjetivo, a la interioridad de cada persona. Tampoco corresponde invocarla respecto de ciertas acciones sanitarias por las personas que ejercen cargos de responsabilidad, sino, por el contrario, deben procurar que no se vulneren los derechos de la población a su cargo, buscando los resguardos para ello.

Podemos concluir que frente a toda objeción de conciencia debe evaluarse si ésta es genuina, asegurándose que no se trate de una supuesta o falsa objeción, motivada por intereses particulares del objetor o que responda a situaciones de discriminación o de poder hacia las poblaciones vulnerables. También debe considerarse que no se trate de una banalización de la libertad de conciencia, simplificando la toma de decisiones, sin efectuar la necesaria reflexión respecto a los argumentos y fundamentos que motivarían una real objeción. La tarea de verificar esta validez podría ser delegada en los comités de ética asistencial, como un mecanismo imparcial. Sin embargo, esta problemática no sólo atañe al círculo establecido entre los involucrados, sino que corresponde tratarla en forma conjunta en la sociedad.
Finalmente, debemos tener presente que la objeción de conciencia en la práctica clínica no puede ni debe ser absoluta, debiendo plantearse en forma prudente, sin imposiciones y sólo como una excepción, toda vez que presenta límites bien definidos, siendo los principales el respeto por la dignidad de todos los involucrados, así como el respeto por el derecho a la vida y a la salud de la población. En la medida en que consideremos la necesidad de compatibilización y respeto por los derechos y libertades fundamentales de cada uno de los integrantes de una sociedad, evitaremos la intolerancia, la discriminación y el conflicto, lo que en definitiva nos permitirá avanzar hacia la mantención de una auténtica democracia, permitiendo que una sociedad con diferentes grupos de ciudadanos, con diferentes valores y creencias puedan aspirar al bien común, tan necesario para la convivencia y el entendimiento.

\section{Agradecimientos}

A Pamela Chávez Aguilar, académica del Departamento de Filosofía, Facultad de Filosofía y Humanidades, Universidad de Chile, por su valiosa colaboración en la revisión de este documento.

\section{Referencias}

1. Associació Catalana D’ Estudis Bioètics (ACEB). Objeción de conciencia en materia de salud. Disponible en http://www.aceb.org

2. Sieira S. La objeción de conciencia sanitaria. Madrid: Editorial Dykinson; 2000: 247.

3. Declaración Universal de los Derechos Humanos. Disponible en http:/www.un.org/es/rights/; www. un.org/es/documents/udhr/

4. Seoane JA. Derecho, ética y salud. Revista Eidon 2006; 22: 11-16.

5. Casas L, Dides C. Objeción de conciencia y salud reproductiva en Chile: Dos casos paradigmáticos. Acta Bioethica 2007; 13(2): 199-206.

6. Martínez K. Medicina y objeción de conciencia. Anales Sistema Sanitario de Navarra 2007; 30(2): 215-223.

7. Cook R, Arango M, Dickens B. Ethical and legal issues in reproductive health. Healthcare responsibilities and conscientious objection. International Journal Of Gynecology And Obstetrics 2009; 104: 249-252.

8. Diccionario de la Real Academia Española. Disponible en http://www.rae.es/rae.html.

9. Abbagnano N. Diccionario de filosofía. México: Fondo de Cultura Económica: 1994: 196-197.

10. Ferrater Mora J. Diccionario de filosofía. Madrid: Editorial Alianza; 1980: 561-562.

11. Canto-Sperber M. Diccionario ética y filosofía moral. Tomo II. México: Fondo Cultura Económica; 2001: 1453 . 
12. Juan Pablo II. Mensaje de S.S. Juan Pablo II para la Jornada Mundial por la Paz de 1991. Dado el 1 de enero de 1991. Biblioteca Electrónica Cristiana. Disponible en http://www.multimedios.org Consultado 23/10/2009.

13. Aparisi A, López J. El derecho a la objeción de conciencia en el supuesto del aborto. De la fundamentación filosófico-jurídica a su reconocimiento legal. Persona y Bioética 2006; 10(1): 35.51 .

14. Figo Committee Report. Ethical Guidelines On Conscientious Objection. Figo Committee for the ethical aspects of human reproduction and women's health. Int Journal Gynec And Obstetrics 2006; 92: 333-334.

15. Domínguez O. Objeción de conciencia, la muerte y el morir en enfermedades en etapa terminal. Acta Bioethica 2009; 15(1): 94-99.

16. Neira K, Szmulewicz E. Algunas reflexiones en torno al derecho general de objeción de conciencia. Revista Derecho y Humanidades 2006; 12: 185-198.

17. Rawls J. Teoría de la Justicia. México: Fondo Cultura Económica; 1993: 391433.

18. Kunstmann M. Desobediencia civil y objeción de conciencia: los límites de la injusticia aceptable. Revista Derecho y Humanidades 1998; 6: 113.126.

19. Savulescu J. Conscientious Objection in Medicine. British Medical Journal 2006; 332(4): 294297.

20. Chervenak F, McCullough L. Conscientious objection in medicine. Author did not meet standards of argument based ethics. British Medical Journal 2006; 332(18).

21. Smith V. Doctor's freedom of conscience. British Medical Journal 2006; 332(18).

22. Murray E, De Zulueta P. The ethics of responding to bird flu. British Medical Journal 2006; 332(18): 425.

23. Araujo J. La objeción de conciencia al servicio militar. Madrid: Editorial Civitas; 1993: 29.

24. Wicclair M. Conscientious Objection in Medicine. Bioethics 2000; 14(3): 205-227.

25. Declaración de la Comisión de Ética y Deontología Médica de la OMC. Objeción de conciencia del médico. Anales del Sistema Sanitario de Navarra 1997; 20(3).

26. Fabó F. Algunas implicancias y problemas bioéticos del diagnóstico prenatal. ARS Médica (Santiago) 2008; 16(16): 147-166.

27. Gómez Fajardo C. Manipulación de embriones humanos: algunas objeciones. IATREIA 2008; 21(1): 75-82.

28. Dickens B, Cook R. The scope and limits of Conscientious Objection. International Journal of Gynecology and Obstetrics 2000; 71: 71-77.

29. Beal M, Cappiello J. Professional Right of Conscience. J Midwifery Womens Health 2008; 53: 406-412.

30. Corte Europea de Derechos Humanos. Pichon and Sajous v. Francia, App. No.49853/99, 2001.

31. Bórquez G. La objeción de conciencia y la responsabilidad médica en la relación clínica. Ponencia en III Seminario Internacional de Bioética "Autonomía del paciente y derecho a decidir". Santiago de Chile: 26-27 junio 2009.

32. Terribas N. Situaciones frontera en la objeción de conciencia: rechazo al tratamientollimitación terapeútica. Ponencia en III Seminario Internacional Bioética "Autonomía del paciente y derecho a decidir". Santiago de Chile: 26-27 junio 2009.

Recibido: 27 de mayo de 2010

Aceptado: 13 de agosto de 2010 